\title{
An adaptive test sequences genera- tion method for the users needs
}

\author{
Richard Castanet \\ Christophe Chevrier \\ Ousmane Koné \\ Bertrand Le Saëc \\ $\mathbf{L} a$ boratoire $\mathbf{B}$ ordelais de $\mathbf{R}$ echerche en $\mathbf{I}$ nformatique \\ 351 crs de la liberation, 33405 Talence cedex, France \\ \{castanet, chevrier, kone, lesaec\}@labri.u-bordeaux.fr
}

\begin{abstract}
We present a test sequences generation method according to users needs. Our method is based on formal languages and monoid computations. The testing users needs are expressed by extended regular expressions. A combination between the specification and the test criteria is computed, providing a specific target sub-specification. Finally, a set of test sequences is generated from this target sub-specification.
\end{abstract}

\section{Keywords}

Conformance testing, formal languages, target sub-specification, faults model, users needs.

\section{INTRODUCTION}

Test sequences generation is an important area of protocol engineering. The generation methods can be applied on the one hand on finite state automata: Distinguishing Sequences [Gon70], W method [Cho78] (and some improvements with Wp [Fa91]), Transition Tour [NT81], Unique Input Output (UIO) sequences [SD85, SD89] (and some improvements with UIOv [VCI89]), on the other hand on Formal Description Technique (FDT) specifications (Estelle, LOTOS ...) [Fav87, CKM92]. The same methods are used with finite state machines and FDT. Empirical methods can be used to produce automatically test sequences. These methods provide sequences similar to those produced by hand by protocols experts [PG90].

All the methods are based on faults model applied to finite state machines (transfer fault between two states, output fault on a transition, $\cdots)\left[\mathrm{BDD}^{+} 91\right]$. The reader can find the presentation for the formal language point of view in [YL95].

The aim of all these methods is to produce the shortest sequences for a type of fault, but these methods make some assumptions for the finite state machine of the specification: determinism, completeness, reset mechanism, equality between the state number of the specification and the implementation... Some extensions have been proposed, for instance, with partially-specified non deterministic state machines [LPB93]. 
The industrial users [CCC92] want sets of test sequences which are accorded to the execution of their applications using the tested protocol. So it appears that their needs do not always correspond to the general behaviour of their application. The conformance sequences are not sufficient enough to verify the behaviour of a protocol in these cases. So, additional sequences have to be built and tested.

We propose the following general goals :

- to consider finite state machine with minimal restrictions (for example without reset mechanism, non deterministic machine, $\cdots$ )

- to take a larger fault model into account (output error, transfer error, additional state, missing state).

- to propose an intensive test of subsets of the specification (parts of the specification considered critical for the application). This approach, that can be named best effort generation, will give a large coverage of the subset, with, of course, the inconvenience of an enlarge set of test sequences.

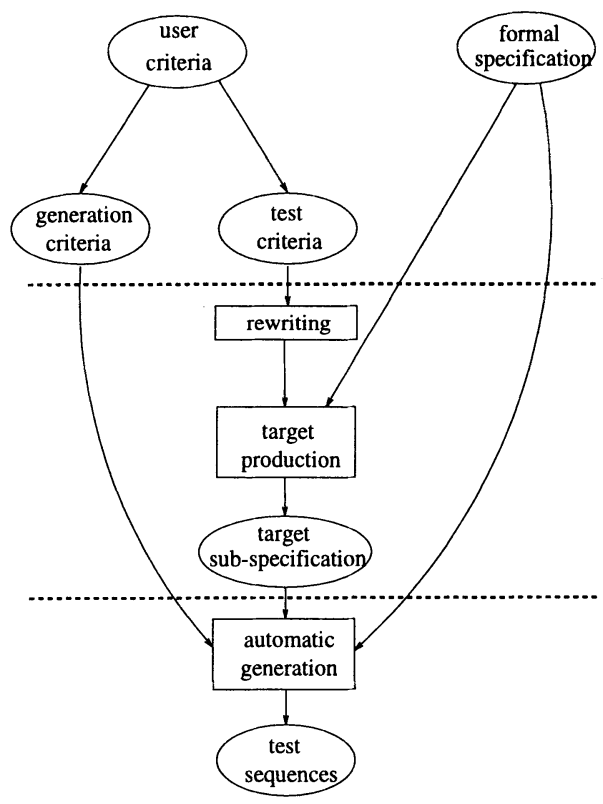

Figure 1 Global architecture of the method

As we use IOSM, we can use formal languages theory and in particular the monoid theory. Each possible calculus in the automaton is associated with an element of a monoid. The considered monoid is finite and interesting elements are the idempotent one. Such an element satisfies the property that the consecutive execution of representatives of this element is equivalent to the execution of ones of them. This is the base of our method and 
we will only consider a finite number of consecutive computations of given idempotents. This method is described in section 4 following the description of the considered fault model in section 3 .

We propose a generation method which is able to cover a large fault model. This method produces a great number of test sequences. So it is not clear to identify the part of the specification which is tested by it. We propose then an overlayer. This tool offers the user the possibility to control the fault model and moreover a criteria specification language. The control of the fault model is based on rules in the monoid to filter the test sequences generation. The criteria specification language is based on generalized regular expressions (predefined operator, references to the states and transitions of the specification). This criteria language correspond to the definition of sub-specifications which are still IOSM. This part is described in section 5 .

The next section is devoted to preliminaries and the last one contains examples.

\section{PRELIMINARIES}

\section{- Regular Expressions}

Let $\Sigma$ be a set of symbols and $\varepsilon$, the empty word . Classical operators with regular expressions are: $\cup$ (union), . (concatenation), * (iteration) and the syntax of an expression $E$ is:

$$
E::=\emptyset|\varepsilon| a|E \cup E| E . E \mid E^{*} \quad \forall a \in \Sigma
$$

$\mathcal{L}(E)$ denotes the language, the set of sequences (or words) over $\Sigma$ associated with $E$, $\mathcal{L}(\emptyset)=\emptyset, \mathcal{L}(\varepsilon)=\{\varepsilon\}, \mathcal{L}(a)=\{a\}, \mathcal{L}\left(E_{1} \cup E_{2}\right)=\mathcal{L}\left(E_{1}\right) \cup \mathcal{L}\left(E_{2}\right), \mathcal{L}\left(E_{1} . E_{2}\right)=\left\{u_{1} . u_{2} \mid u_{1} \in\right.$ $\left.\mathcal{L}\left(E_{1}\right), u_{2} \in \mathcal{L}\left(E_{2}\right)\right\}, \mathcal{L}\left(E^{*}\right)=\{\varepsilon\} \cup\left\{u_{1} \ldots u_{n} \mid \forall 1 \leq i \leq n, u_{i} \in E\right.$ and $\left.n \geq 0\right\}$.

- Automata

Let $\Sigma$ be a finite alphabet ( $\Sigma$ is a set of input and output symbols). $\Sigma^{+}$denotes the words built over the alphabet $\Sigma$ and $\Sigma^{*}=\Sigma^{+} \cup\{\varepsilon\}$. An automaton $\mathcal{A}$ is a quadruple $\left(Q, q_{0}, \delta, F\right)$ where $Q$ is a finite set of states, $q_{0}$ the initial state, $\delta$ the transfer function: $Q \times \Sigma \rightarrow Q, F$ is the set of final states, $F \subset Q . \mathcal{L}(\mathcal{A})$ denotes the language recognized by the automaton $\mathcal{A}$. A word $w \in \Sigma^{*}$ belongs to $\mathcal{L}(\mathcal{A})$ if and only if $q_{0} \stackrel{w}{\longrightarrow} q_{f}$ where $q_{f} \in F$. Figure 6 depicts an automaton which specifies a part of the INRES protocol.

- The transitional monoid [Ber79, Eil76, Lal79, Pin86]

With any automaton $\mathcal{A}\left(Q, q_{0}, \sigma, F\right)$, we can associate a congruence $\approx$ defined on $\Sigma^{*}$ (i.e., an equivalence relation $\approx$ satisfying: $u, v \in \Sigma^{*}, u \approx v \Rightarrow \forall w, w^{\prime} \in \Sigma^{*}, w u w^{\prime} \approx w v w^{\prime}$ ) in order to describe the computation in $\mathcal{A}$ in a global way:

$$
\forall u, v \in \Sigma^{*}, u \approx v \text { iff } \forall q, q^{\prime} \in Q,\left(q \stackrel{u}{\rightarrow} q^{\prime}\right) \Leftrightarrow\left(q \stackrel{v}{\rightarrow} q^{\prime}\right)
$$

This congruence, named the transitional congruence of $\mathcal{A}$, is of finite index. $u_{\approx}$ denotes the class of $u$, i.e. the set of words equivalent to $u$. Two different words in a same class have same computations from any state $q$ to a same state $q^{\prime}$ of $\mathcal{A}$. In the example of the figure 2 , the congruence $\approx$ has 4 classes:

$$
\varepsilon_{\approx}=\varepsilon, a_{\approx}=a^{+}, b_{\approx}=b^{+}, a b_{\approx}=a^{+} b \Sigma^{*} \cup b^{+} a \Sigma^{*} .
$$




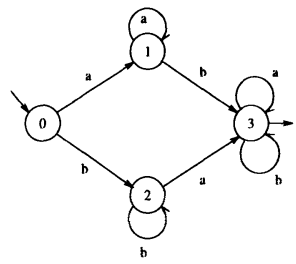

Figure 2 Example of Finite State Machine

\begin{tabular}{|c||c|c|c|c|}
\hline$\circ$ & $m_{\epsilon}$ & $m_{a}$ & $m_{b}$ & $m_{a b}$ \\
\hline \hline$m_{\epsilon}$ & $m_{\epsilon}$ & $m_{a}$ & $m_{b}$ & $m_{a b}$ \\
\hline$m_{a}$ & $m_{a}$ & $m_{a}$ & $m_{a b}$ & $m_{a b}$ \\
\hline$m_{b}$ & $m_{b}$ & $m_{a b}$ & $m_{b}$ & $m_{a b}$ \\
\hline$m_{a b}$ & $m_{a b}$ & $m_{a b}$ & $m_{a b}$ & $m_{a b}$ \\
\hline
\end{tabular}

Table 1: Composition table

With any congruence $\approx$, we can associate a "transitional monoid" $\mathcal{M}$ (i.e. a set equipped with an associative operation denoted by $\circ$ and a neutral element $\left.\epsilon_{\approx}\right)$ in bijection with the set of classes of $\approx$. In order to clarify the presentation, we denote by $m_{u}$, the element of $\mathcal{M}$ in bijection with $u_{\approx}$. For our example, the monoid is defined by $\mathcal{M}=\left\{m_{\epsilon}, m_{a}, m_{b}, m_{a b}\right\}$

The operation $\circ$ associated with $\mathcal{M}$ is defined by: $m_{u} \circ m_{v}=m_{u v}$. We can also define a composition table of the elements of $\mathcal{M}$ (Table 1 for the previous monoid).

If the congruence is of finite index, the monoid $\mathcal{M}$ is obviously finite. So we have a finite representation of a language (the one recognized by $\mathcal{A}$ ) which has an infinite number of words.

The test sequences generation method, we propose in the following, is closely linked to a notion of "strong periodicity". In monoid theory, this notion can be found in the notion of idempotence:

$$
f \in \mathcal{M} \text { is an idempotent if } f \circ f=f^{2}=f
$$

The neutral element of a monoid is always an idempotent and if the monoid has a zero, it is also an idempotent.

Any element of a finite monoid admits a power which is an idempotent (see, for instance, $[\operatorname{Pin} 86])$ :

Theorem 2.1 For all finite monoid $\mathcal{M}, \exists k_{\mathcal{M}} \in \mathbb{N}$ such that $\forall m \in \mathcal{M}$, we have $m^{k_{\mathcal{M}}}=$ $m^{2 k_{\mathcal{M}}}$.

The number $k_{\mathcal{M}}$ is characteristic of the monoid, it does not depend on the considered element $\mathrm{m}$.

We also use the famous Ramsey theorem [GRS80]:

Theorem 2.2 Let $E$ be a set, we denote $P_{p}(E)$ the set of subset of $E$ with p elements. Let $\theta$ be a partition of $P_{p}(E)$ in $k$ classes. If, for any $n$, there exist $R(n, p, k)$ such that $\operatorname{Card}(E) \geq R(n, p, k)$ then there exist a subset $F \subset E$ with $n$ elements such that $P_{p}(F)$ is included in a class of $\theta$.

Applying this result to the computation of sequences in the monoid $\mathcal{M}$ having $k$ elements (with $p=2$ and $n=n^{\prime} * k_{\mathcal{M}}$ ), we can easily obtain the following corollary: 
Corollary 2.3 For any finite monoid $\mathcal{M}$ with $k$ elements, there is $R\left(n^{\prime} k_{\mathcal{M}}, 2, k\right) \in \mathbb{N}$ such that any sequence longer than $R$ can be factorized in $n^{\prime}+2$ parts:

$$
s: m_{1} \underbrace{m^{k_{\mathcal{M}}} \ldots m^{k_{\mathcal{M}}}}_{n^{\prime}} m_{2}
$$

where, according to theorem $2.1, m^{k_{\mathcal{M}}}$ is an idempotent of the monoid $\mathcal{M}$ and $m_{1} \circ m^{k_{\mathcal{M}}}=$ $m_{1}$.

\section{FAULT DETECTION POWER}

\subsection{Protocols as automata}

Specifications of protocols are represented by inputs outputs state machines (IOSM). In these machines, the alphabet is the union of input alphabet, output alphabet and internal alphabet (see example in figure 6). IOSM can always be reduced in a deterministic minimal automata. Our tool tool contain an algorithm wich provide minimal automata. So in the sequel we suppose that the used automata are deterministic and reduced. Moreover protocols modelize iterations of periodical prefix processes. So, in the sequel, we also assume that the used automata are strongly connected (with eventually an additional sink state) and they have only one final state which is the initial one.

\subsection{Faults model}

Several types of implementation faults linked to specification modelized by IOSM can be defined [BDD $\left.{ }^{+} 91\right]$. We have mainly two basic faults: "output faults" and "transfer faults" (respectively bad output event and bad final state for a transition of the automaton). Moreover, some other faults can be considered: missing state, missing transition and additional state or transition.

Generally, in order to express the covered fault model, the generation method assumes that the tested implementation is complete and has the same number of states than the implementation. In this case the fault model is only composed by the basic faults.

In our approach, we make also supose that the implementation is complete, but we do not need any assumptions on the size of the implementation. So we have to take into account two other types of faults:

- "Simple additional state fault": An additional state is considered as a fault if the calculus from it in the specification do not correspond to the ones from any states of the specification. For the specification described in the figure 3 , the $b$ ) is a correct implementation of $a$ ) but $c$ ) is a faulty one (the transition $\left(3^{\prime}, e, 5\right)$ is omitted.

- "Linearization fault": The others additional state type of faults are due to linearizations of loops. A n-linearization of a loop is the substitution of this loop by $n$ consecutive paths with the same label. For example, figure 4 a points out a specification that contains a loop on the state 1 . The implementation of figure $4 \mathrm{~b}$ is a correct one but the implemention $4 \mathrm{c}$ and $4 \mathrm{~d}$ are faulty ones due to a 1-linearization, respectively a 2 -linearization, of the loop $b$.

The method described in the sequel allows to capture, in a certain limit, this fault model. This limit is completely controled by the user. 


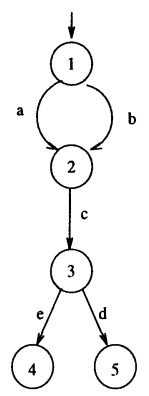

a)

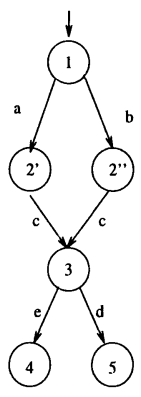

b)

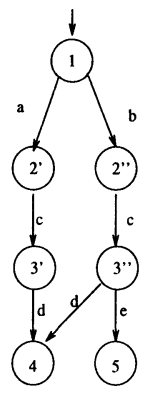

c)

Figure 3 simple additional state fault

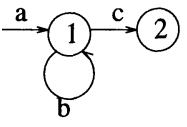

a)

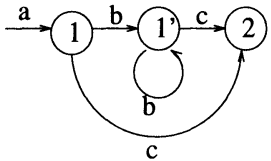

b)

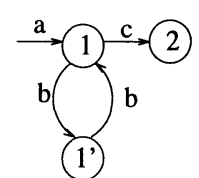

c)

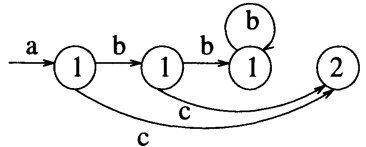

d)

Figure 4 linearization fault

\section{Test sequences generation}

The basic method, we enlarge here, has been described in [CCSC95]. It can be viewed as a general method that incrementally develops sequences for a given goal (initial state to final one). The sequences are kept or rejected with respect to the generation rule that consider the global behaviour of the transitions in the specification.

\subsection{Application to communication protocol test}

In a "well written" specification, two elements of the alphabet have different behaviours; i.e. their transfer functions on the automaton are different. So, in the transitional monoid of a specification, two different elements of the alphabet correspond to two different elements. We call basic element, an element of the monoid which corresponds to a letter of the alphabet.

For the transitional monoid, the idempotent property $f_{u}^{2}=f_{u}$ means that:

$$
\forall u^{\prime}, u^{\prime \prime} \in u_{\approx}, \forall q \in Q, \delta\left(q, u^{\prime}\right)=q^{\prime} \Rightarrow \delta\left(q^{\prime}, u^{\prime \prime}\right)=q^{\prime}
$$

Our method consist in controlling the number $n^{\prime}$ of consecutive iterations of a same idempotent: For any $n^{\prime} \geq 0$, we will consider only factorization of $m \in \mathcal{M}$ of the form $\left(\alpha, f^{i}, \beta\right)$ where $0 \leq i \leq n^{\prime}-1$ with $\alpha, f, \beta \in \mathcal{M}, \alpha \neq f, \beta \neq f, f^{2}=f, \alpha f=\alpha$. This set of factorization is finite. So for each $n^{\prime}$, Corollary 2.3 guaranties that the set of sequences, obtained by factorization of an element of the monoid, is finite. 
We first introduce the method on the example of the figure 2. The first step of the method leads us to calculate the classes of the congruence $\approx$ and the composition table of the corresponding monoid ( $\mathrm{cf}$ table 1 ). In this case, we want to produce some sequences which lead form $q_{0}$, the initial state to $q_{3}$, the final one. The corresponding element in the monoid is $m_{a b}$ so the sequences will be deduced from the factorization of this element. For $n^{\prime}=3$, the obtained set of factorization is:

$$
\begin{aligned}
& m_{a b} \rightarrow\left\{m_{a b},\right. \\
& m_{a b} m_{a b}, m_{\varepsilon} m_{a b}, m_{a b} m_{\varepsilon}, m_{a} m_{b}, m_{b} m_{a}, \\
& \left.m_{\varepsilon} m_{\varepsilon} m_{a b}, m_{a} m_{a} m_{b}, m_{b} m_{b} m_{a}, \ldots\right\}
\end{aligned}
$$

In this example, all the elements of the monoid are idempotent and so no generated factorization contains terms like $m_{\varepsilon}^{3}, m_{a}^{3}, m_{b}^{3}, m_{a b}^{3}$. Then, we replace the elements of the monoid by their representatives in the alphabet. We obtain, extracting from this set all the sequences that are prefixes of another the following set of sequences:

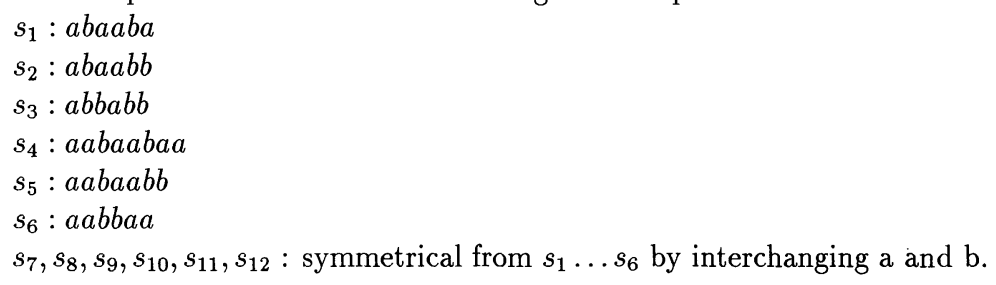

\subsection{Sequences generation algorithm}

The monoid associated with a specification is computed using the AMORE system (computing Automata, MOnoid, and Regular Expression)[JPTW90], developed at the Aachen university since 1986, is a tool for finite states automata, syntactic monoids [Pin86][Eil76] and regular languages computations. It offers different procedures that convert regular expressions or automata into complete deterministic minimal automata, moreover it compute the associated syntactic monoids. The syntactic monoid is the transitional monoid of the minimal automaton.

We select the set of elements which correspond to calculus from the initial state to itself and we only develop test sequences for this set of elements. So, we obtain sequences which can be chained with no need of a reset.

Each class admits a factorization (cf table 1) whose first term is a basic element. We take into account only such factorizations. We compute in an incremental way (developing at each step the left term of the factorization of a class) the test sequences $\sigma$ verifying the property:

Generation rule: If a sequence $\sigma$ is factorizable in the form:

$$
\begin{aligned}
& \sigma=a_{1} \ldots a_{i_{1}} a_{i_{1}+1} \ldots a_{i_{2}} a_{i_{2}+1} \ldots a_{i_{3}} a_{i_{3}+1} \ldots a_{i_{n^{\prime}}} a_{i_{n^{\prime}}+1} \ldots a_{j} \\
& \quad \text { with } f=\left(a_{i_{1}+1} \ldots a_{i_{2}}\right)=\ldots=\left(a_{i_{n^{\prime}-1}+1} \ldots a_{i_{n^{\prime}}}\right) \\
& \text { then } f^{2} \neq f .
\end{aligned}
$$

(i.e. not any chosen sequences contain $n^{\prime}$ consecutive identical idempotent factors.)

We refine the previously built set $T_{1}$, avoiding any sequence of $T_{1}$ which is strict prefix (operator $\leq$ ) of another one in $T_{1}$. So we produce the set $T_{2}$ : 


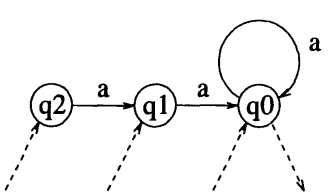

a)

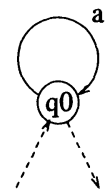

b)

Figure 5 Example of behaviour lost

Prefix rule: $\quad \sigma \in T_{2}$ iff:

- $\sigma \in T_{1}$

- $\forall \sigma, \sigma^{\prime} \in T_{2}, \sigma \leq \sigma^{\prime} \Rightarrow \sigma=\sigma^{\prime}$

- $\forall \sigma^{\prime} \in T_{1}, \exists \sigma \in T_{2}$ such that $\sigma^{\prime} \leq \sigma$

In fact, the implementation of our algorithm directly works on the words corresponding to basic elements.

Corollary 2.3 guaranties the termination of the algorithm and so, our set of sequences is finite.

\subsection{Generating from a sub-specification}

In the following section we give the user the possibility to express a sub-specification (i.e. a part of the initial specification). We have seen that a monoid is able to express some properties in a global way. When we have a sub-specification, some informations about the global behaviour of some inputs or outputs are lost. Let us consider the figure 5 that describes two parts $(a)$ and $b$ ) ) of a same specification where transition labelled by " $a$ " appears. In the complete specification, the sequence " $a a$ " corresponds to an idempotent, it is not the case for the sequences " $a$ ". If we consider a sub-specification where only the configuration b) appears, then the basic element " $a$ " becomes an idempotent. If we directly extract sequences from this sub-specification, factors "aaa" and "aaaa" are not allowed. But in the complete specification, this factors may occurs in the test sequences. So, in this case we have lost an information about the behaviours of the transitions labelled by " $a$ " in the sub-specification.

We have to take into account both the monoid which corresponds to the target specification and the monoid which corresponds to the total specification in our test sequence generation. The first monoid is used to develop the sequences and the second one to compute the possible reductions of the sequences.

\section{USER CONTROL}

In this section, we propose to the user two ways to control the generation of the test sequences. The first control concerns the possibility to define targets to test in the specifications. In order to obtain this, we propose a language to manipulate test criteria. The 
second allows the user to increase or decrease the fault model. A convenient interface will be provided in the sequel.

\subsection{Formal expression of test criteria}

The aim of the section is to provide a formal way to express test criteria. A criterion is a user requirement for a particular test. A criterion being defined, our method selects a set of sequences that matches the criterion.

As we use automata theory, regular expressions (see below) will be the basis to express test criteria.

\subsubsection{User Requirements}

Let $\left(\Sigma, Q, q_{0}, \delta, F\right)$ be an automaton specification. The automaton is defined with some "characteristic objects" that are

- the set of states,

- the symbols from the alphabet,

- the transfer function or the transitions.

It is obvious that user requirements for a particular test are defined over these objects.

(1) Testing a particular transition is a widely used requirement (see the survey by [SL89]). Such a test consists of executing some preamble which is a sequence that leads the IUT (Implementation Under Test) to the origin of the transition, executing the transition, and eventually executing some postamble (the sequence after the transition). This is also known as the "single transition" test method and it is basically used in many tests requirements. For instance,

(2) Testing a transition with all possible preambles,

(3) Testing a given sequence of transitions

(4) Performing the previous test (3) $n$ consecutive times $(n=2,3, \ldots)$.

We consider the automaton specification of figure 6 and the previous requirements are used in the sequel to illustrate the proposed formalism for test criteria.

\subsection{2 (Test) Criteria Expressions}

The reader may report to [Aut87, Pin86, BS86] for more details on regular expressions.

A test criterion is defined by an expression. We define a test criterion expression or simply criterion expression $(C E)$ by means of (additional) operators that are more suitable to facilitate criteria description and to improve the readability. These operators are $+, *, \wedge, \cap, \cup,-$, LENGTH, length, MIN, min, NOLOOPS.

$E$ stands for a regular expression, $C E$ stands for a criterion expression, $q_{k}$ stands for state $k$ and $i, j, n$ stand for integers.

The expressions syntax is defined in a recursive way by the grammar:

$$
\begin{aligned}
& C E::=\left(q_{0} S\right)\left|C E^{+}\right| C E^{*}|C E \wedge n|(C E \cap C E)|(C E \cup C E)|(C E-C E) \\
& S::=F q_{k} S \mid F . q_{0} \quad \forall k \in Q \\
& F::=M I N(E)|\min (E)| N O L O O P S(E) \mid E \\
& E::=\emptyset|\varepsilon| a|E \cup E| E . E\left|E^{*}\right| E^{+}|\operatorname{LENGTH}(E, i, j)| \text { length }(E, i, j)
\end{aligned}
$$


The grammatical rule $C E$ acts as a generator and a combiner of criterion expressions. The rule $S$ permits to describe the general structure of a criterion expression. The operation on the extended regular expressions are defined by the rule $F$ and extended regular expressions by the rule $E$.

The criteria described by users are combinations of criterion $C E$ in the following form:

$$
q_{0} \cdot F_{1} \cdot q_{i_{1}} \cdot F_{i_{2}} \cdot q_{2} \cdots F_{n} \cdot q_{0}
$$

by means of the operators $+, *, \wedge, \cap, \cup,-$ whose semantic is:

- $\mathcal{L}(C E *)=\mathcal{L}(C E)^{*}$

$*$ defines iteration.

- $\mathcal{L}(C E+)=\mathcal{L}\left(C E^{*}\right)-\{\varepsilon\}$

+ defines iteration without the empty word, $\varepsilon$.

- $\mathcal{L}\left(C E_{1}-C E_{2}\right)=\mathcal{L}\left(C E_{1}\right)-\mathcal{L}\left(C E_{2}\right)$

- defines the difference of the 2 languages associated with 2 criterion expressions.

- $\mathcal{L}(C E \wedge n)=\mathcal{L}(C E . C E \ldots C E) n$ times

$\wedge$ (power) defines the number of iterations of a criterion expression.

- $\mathcal{L}\left(C E_{1} \cap C E_{2}\right)=\mathcal{L}\left(C E_{1}\right) \cap \mathcal{L}\left(C E_{2}\right)$

$\cap$ defines the intersection of the 2 languages associated with 2 criteria expressions.

- $\mathcal{L}\left(C E_{1} \cup C E_{2}\right)=\mathcal{L}\left(C E_{1}\right) \cup \mathcal{L}\left(C E_{2}\right)$

$\cup$ defines the union of the 2 languages associated with 2 criteria expressions.

The language associated with a criterion expression $C E$ of the form:

$$
C E=\left(q_{0} \cdot F_{1} \cdot q_{i_{1}} \cdot F_{2} \cdot q_{i_{2}} \cdots F_{n} \cdot q_{0}\right)
$$

is equal to:

$$
\mathcal{L}\left(q_{0} \cdot F_{1} \cdot q_{i_{1}}\right) \cdot \mathcal{L}\left(q_{i_{1}} \cdot F_{2} \cdot q_{i_{2}}\right) \cdots \cdot \mathcal{L}\left(q_{i_{n-1}} F_{n} \cdot q_{0}\right)
$$

where $F_{i}$ is $M I N(E), \min (E), N O L O O P S(E)$ or simply an extended regular expression $\mathrm{E}$ (using length). Each language $\mathcal{L}\left(q_{i} . F_{i} . q_{j}\right)$ describes the words defined by $F_{i}$ which lead from $q_{i}$ to $q_{j}$. In particular, $\mathcal{L}\left(q_{i} . S . q_{j}\right)=\mathcal{L}_{i j}$ denote the set of word $u$ in the specification such that $q_{i} \stackrel{u}{\longrightarrow} q_{j}$

- $\mathcal{L}\left(q_{i} . E . q_{j}\right)=\mathcal{L}_{i j} \cap \mathcal{L}(E)$

- $\mathcal{L}\left(q_{i} . M I N(E) . q_{j}\right)=\left\{u \in \mathcal{L}\left(q_{i} . E . q_{j}\right)\left|\forall v \in \mathcal{L}\left(q_{i} . E . q_{j}\right),\right| u|\leq| v \mid\right\}$ where $|u|$ is the length of $u$

$M I N$ defines the set of words of an extended regular expression with minimal length.

- $\left.\mathcal{L}\left(q_{i} \cdot \min (E) . q_{j}\right)=u \in \mathcal{L}\left(q_{i} \cdot E \cdot q_{j}\right)\left|\forall v \in \mathcal{L}\left(q_{i} \cdot E . q_{j}\right),\right| u|\leq| v \mid\right\}$

$\min$ is a word of an extended regular expression with a minimal length. 
- $\mathcal{L}\left(q_{i} . N O L O O P S(E) . q_{j}\right)=\left\{u \in \mathcal{L}\left(q_{i} . E . q_{j}\right)\left|\forall v w v^{\prime} \in \Sigma^{+}\right| u=v w v^{\prime}, q_{i} \stackrel{v}{\rightarrow} q_{j} \stackrel{w}{\rightarrow} q_{k} \stackrel{v^{\prime}}{\rightarrow}\right.$ $\left.q_{f} \Rightarrow q_{j} \neq q_{k}\right\}$

NOLOOPS denotes the set of words of an extended regular expression which do not use loops.

- $\mathcal{L}(L E N G T H(E, n, m))=\{u \in \mathcal{L}(E)|n \leq| u \mid \leq m\}$

$L E N G T H$ denotes the set of words of an extended regular expression with a specified length.

- $\mathcal{L}($ length $(E, n, m))=u \in \mathcal{L}(E)|n \leq| u \mid \leq m$

length denotes a word of an extended regular expression with a specified length.

In next section, we give some examples of criteria expressions that use these operators.

\section{Criteria Expression Examples}

(1) $C E_{1}=q_{0} \cdot \Sigma^{*} \cdot q_{5} . I D A T r e q . \Sigma^{*} \cdot q_{0}$ send an IDATreq in state $q_{5}$ with any preamble and any postamble.

(2) $C E_{2}=q_{0} \cdot \min \left(\Sigma^{*}\right) \cdot q_{5} . I D A T r e q . \Sigma^{*} \cdot q_{0}$ same as (1) but with a preamble of minimal length.

(3) $C E_{3}=q_{0} \cdot \Sigma^{*} \cdot q_{5} \cdot\left(\right.$ ID ATreq. $\left.\Sigma^{*}\right) \wedge 3 . q_{0}$ brings the IUT in state $q_{5}$ with any preamble, then send an IDATreq followed by some postamble, 3 consecutive times.

(4) $C E_{4}=q_{0} \cdot \Sigma^{*} \cdot q_{5} \cdot L E N G T H\left(I D A T r e q . \Sigma^{*}, 3,5\right) \cdot q_{0}$ brings the IUT in state $q_{5}$ with any preamble, then sends a sequence starting with an IDATreq which length is between 3 and 5.

\subsubsection{From test criteria expression to target sub-specification}

It is not difficult to translate the test criteria expressions into regular expressions and then to combine the criteria with the specification in order to obtain the part of the specification that the user want to test (the target sub-specification). The first step of the method allows to transform all the additional operators used by the criteria. In the second step, we eliminate the state notation to merge the alphabet of the specification.

When the criterion expression has been transformed in the form of a regular expression the final step, before the generation step, consists of the generation of the target subspecification. It corresponds to the computation of the intersection between the criteria expression and the specification. If the intersection is empty, then it means that the expressed criteria is not suitable with the specification.

All the operations over automata and regular expressions we made are computed using the software AMORE [JPTW90].

\subsection{Formal expression of the fault model}

Basicaly, the generation method works with $n^{\prime}=3$. In this case, the set of sequences contains a distinguishing sequences if it exists, the UIO sequences if they exist, a set of 
caracterisation as produce by the $\mathrm{W}$ method. Moreover, we can test all the faults due to a 1-linearization of loops and the faults due to simple additional state fault. The user can modify this model either increasing or decreasing it

\subsubsection{Test weakness}

We can reduce the number of sequences and so decrease the fault detection power by adding some test rules. For example, the user can specify the following rules:

Orderness rule: If two sequences $S_{1}$ et $S_{2}$ are built in the following form:

$$
\begin{aligned}
& S_{1}: a_{0} \ldots a_{h} \ldots a_{i} a_{i+1} \ldots a_{j} a_{j+1} \ldots a_{g} a_{g+1} \ldots a_{p} \\
& S_{2}: a_{0} \ldots a_{h} \ldots a_{i} a_{j+1} \ldots a_{g} a_{i+1} \ldots a_{j} a_{g+1} \ldots a_{p}
\end{aligned}
$$

with $m=\left(a_{h} \ldots a_{i}\right)_{\approx}, f=\left(a_{i+1} \ldots a_{j}\right)_{\approx}, g=\left(a_{j+1} \ldots a_{g}\right)_{\approx}$ and $m \circ g=m$ and $m \circ f=m$ then we only keep one of them.

This rule intuitively expresses that the order of loops appearance is not important in this case.

Repetition rule: If a sequence $\sigma$ is built in the following form:

$$
\sigma: a_{0} \ldots a_{h} \ldots a_{i} a_{i+1} \ldots a_{j} a_{j+1} \ldots a_{g} a_{g+1} \ldots a_{p}
$$

with $m=\left(a_{h} \ldots a_{i}\right) \approx, f=\left(a_{i+1} \ldots a_{j}\right) \approx=\left(a_{j+1} \ldots a_{G}\right) \approx, m \circ f=m$ and $f \circ f=f$ then we only keep one $f$ factor.

This rule expresses that a sequence never contain two consecutive idempotent factors. In particular, a loop which belongs to an idempotent class is tested only once.

These reduction rules can be expressed in a global way or in a local way (specifying the elements on which the rules can be applied).

Comments: These reduction rules do not garantie the basic fault model.

\subsubsection{Test increase}

The basic method is able to detect the faulty implementation of figure $4 c$ ( " $b$ " correspond to an idempotent), but it cannot detect the faulty implementation of figure $4 \mathrm{~d}$ due to a 2-linearization. Intuitively, this detection is linked to the number of iterations of the faulty loop specified in the generation rule. If we enforce the generation rule, we are able to detect faults hide by $n$-linearizations. It correspond to the parametrization of the generation rule by $n^{\prime}: m f^{n^{\prime}+1} \longrightarrow m f^{n^{\prime}}$ with $m \circ f=m$ and $f \circ f=f$.

\subsection{Advantages of the method}

\subsubsection{Industrial needs in communication protocol testing}

We can mention the following requirements:

- Each sequence has to begin in the initial state and return to the initial state (this is the usual representation of a test sequence obtained in an empirical way).

- To lead the specification to a given state and to go to another given state to cover a part of the specification. 
- To have a large choice of sequences selections to cover given areas of the specification.

- To maximize the number of possible testable errors.

\subsubsection{Advantages of the sequences generated with our method}

- Production of sequences whatever required initial and final states, and in particular the possibility to produce sequences for inopportune events: If the specification has an error state where leads all an inopportune event transition and a reset to return to the normal behaviour, the general method produce some sequences to test these transitions. But if the error state is a sink state, we have to produce unchainable sequences which lead from $q_{0}$ to the sink state.

- Ability of criteria expression by means of the test criteria languages.

- Taking into account of the required faults model by means of the generation rules (orderness, repetition rules $\cdots$ ).

- Preservation of the fault detection power of the sequences even if they are not applied to the initial state. For instance, if the IUT is not in its initial state, the used sequences are still valid.

\section{EXAMPLES}

We illustrate our generation method on the example of the INRES protocol [Hog92]. This protocol provide a simple asymmetric data transfer over an unreliable medium. The connection asked by the initiator is established by means of the primitives: ICONreq, ICONind, ICONres, ICONconf and then the data transfer by the primitives IDATreq and IDATind. At any time the connection can be disrupted by the responder by means of the primitives IDISreq and IDISind. A specification for the initiator part is given figure 6 . We give below some examples of criteria and the corresponding sequences for this specification. We use for these examples a local test architecture.

To this automaton corresponds a monoid with 1198 elements. If we apply our method on this monoid, the produced set of sequences will be too big to be used to test the implementation. Also, with the test criteria expression language, we cut the test problem in different test objectives. The examples below are inspired from the basic test of the 9646 standard [ISO].

- Test purpose: Basic connection test - this test aims at verifying if the implementation under test (IUT) is able to connect to another entity. The criterion is expressed as follows:

$$
C E_{\text {basic_connection }}=q_{0} \cdot I C O N r e q .(\Sigma-(I D A T r e q \cup T 1))^{*} . I D I \text { Sind } . q_{0}
$$

This criterion means that the upper tester asks for a connection (ICONreq) and waits for a connection end (IDISind). Not any data transfer (IDATreq) or reconnection timer $(T 1)$ are authorized at this level of test.

The monoid which corresponds to the intersection with the specification have 38 elements. The test set is composed by only 4 sequences. 


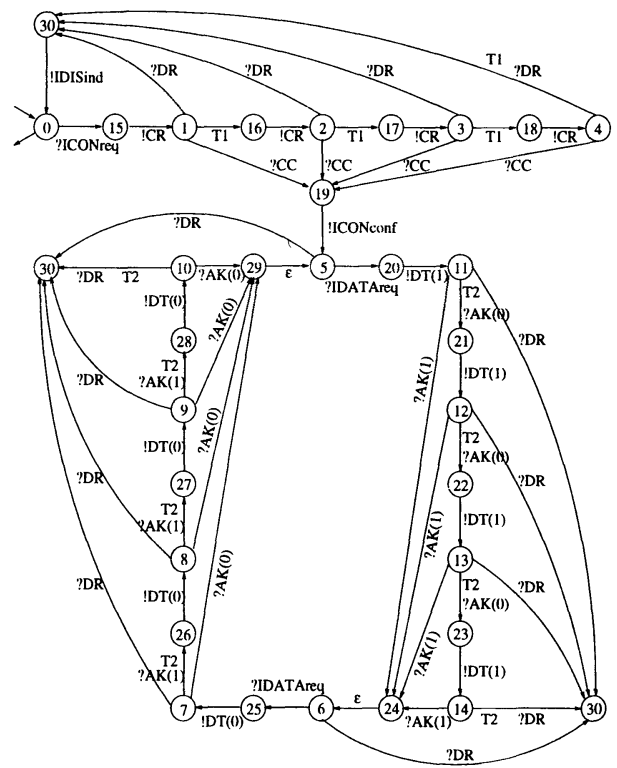

Figure 6 IOSM for the initiator part of the InRes protocol

ICOHreq. CR.DR. IDIS ind. ICOHreq.CR.DR. IDIS ind

ICOIIreq.CR.DR. IDIS ind. ICOIreq.CR.CC. ICOIIconf.DR. IDISind

ICOIreq.CR.CC. ICOIIconf. DR. IDIS ind . ICOIreq.CR. DR. IDIS ind

ICOIreq. CR. CC. ICOIIconf. DR. IDIS ind. ICOIreq. CR . CC. ICOIconf. DR. IDIS ind

- Test purpose: Elementary data transfer - this test aims at verifying the ability of the IUT to exchange data with another entity. The criterion is expressed as follows:

$$
C E_{\text {elementary_data_transfer }}=q_{0} \cdot \min \left(\Sigma^{*}\right) \cdot q_{5} \cdot I D A T r e q \cdot q_{20} \cdot \min \left(\Sigma^{*} \cdot A K(1) \cdot \Sigma^{*}\right) \cdot q_{0}
$$

This criterion means that we want to take a minimal way to lead the implementation in the state $\left(q_{5}\right)$ in which data transfers are allowed. Then we asked for a data transfer (IDATreq) and we terminate the connection in the shortest way with a positive acknowledgement.

The corresponding monoid have 83 elements and it allows us to produce one sequence:

ICOIreq.CR.CC. ICOIConf . IDATreq.DT(1) . AR (1) . DR .IDISind .

ICOIreq.CR.CC.ICOIconf. IDATreq. DT (1). AK (1).DR.IDIS ind

- Test purpose: Behaviour with respect to connection timer - this test aims at verifying the connection protocol with respect to the connection timer. The criterion is expressed as follows:

$C E_{\text {connection_behaviour }}=q_{0} . I C O N r e q \cdot(\Sigma-(D R))^{*} \cdot I C O N$ conf.$(\Sigma-(\text { IDATreq }))^{*} \cdot q_{0}$ 
This criterion means that, after a connection request, (ICONreq) all events, and in particular the event corresponding to a connection timer end, are authorized except a disconnection request (DR). After a connection confirm (ICONconf), not any data transfer (IDATreq) is accepted.

The target sub-specification is associated with a monoid which contains 155 elements and we produce a set of 16 sequences:

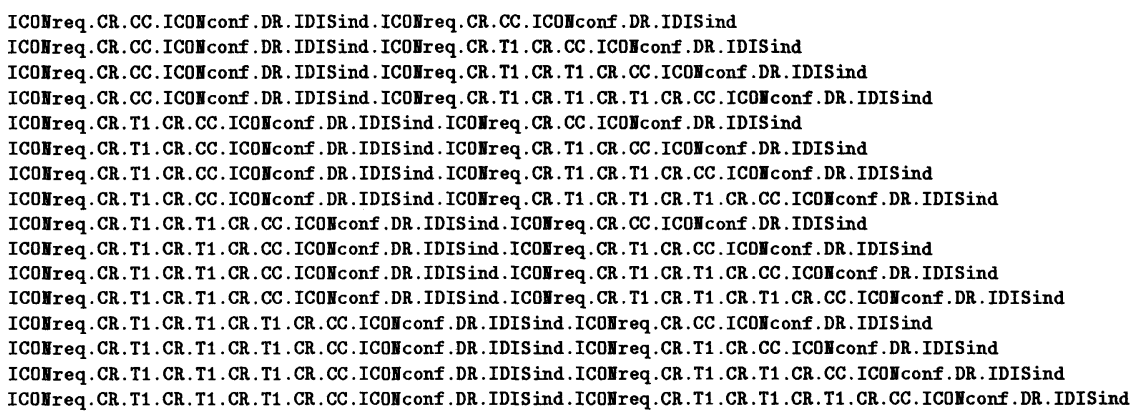

\section{REFERENCES}

[Aut87] J.M. Autebert. (Algebraic Langages) Langages Algébriques. Etudes et Recherches en Informatique. Masson, 1987.

[BDD ${ }^{+91}$ G.v. Bochmann, A. Das, R. Dssouli, M. Dubuc, A Ghedamsi, and G Luo. Fault models in testing. In $4^{\text {th }}$ IWPTS, Leishendam, The Netherlands, october 1991.

[Ber79] J. Berstel. Transductions and context free Languages. Terbner Studienbucher, Stuttgart, 1979.

[BS86] G. Berry and R. Sethi. From regular expressions to deterministic automata. Theoritical Computer Science, (48):117-126, 1986.

[CCC92] R. Castanet, R. Casadessus, and P. Corvisier. Industrial experience on test suite coverage management. In $5^{\text {th }}$ IWPTS, Montréal, Canada, october 1992.

[CCSC95] C. Chevrier, R. Castanet, B. Le Saëc, and R. Casadessus. Génération de séquences de test par calcul de monoïdes. In CFIP'95, Rennes, France, may 1995. Hermes.

[Cho78] T.S. Chow. Testing software design modelled by finite state machines. IEEE Transactions on software engineering, SE-4(3):178-187, may 1978.

[CKM92] A. Cavalli, S. Kim, and P. Maigron. Automated protocol conformance test generation based on formal methods for lotos specifications. In $5^{\text {th }}$ IWPTS, Montréal, Canada, october 1992.

[Eil76] S. Eilenberg. Automata, langages and machines, volume A, B. Academic Press, New York, 1974-1976,.

[Fa91] S. Fujiwara and all. Test selection based on finite state models. IEEE Transaction on software engineering, 17(6), june 1991.

[Fav87] J. P. Favreau. Automatic generation of test scenario skeletons from protocol specifications written in estelle. In $6^{\text {th }}$ PSTV. North Holland, 1987.

[Gon70] G. Gonenc. A method for the design of fault detection experiment. IEEE Transaction on computer, C-19(6), june 1970. 
[GRS80] Ronald L. Graham, Bruce L. Rothschild, and Joel H. Spencer. Ramsey theory. Wiley interscience series in discrete mathematics. John Wiley and Sons, 1980.

[Hog92] D. Hogrefe. Osi formal specification case study: the inres protocol and service, revised. Universität Bern", 1992.

[ISO] ISO. Information processing systems - open systems interconnection - conformance testing methodology and framework- part 1: general concept - part2: abstract test suite specification - part3: the tree and tabular combined notation (ttcn) - part4: test realization - part5: requirement on test laboratories and clients for the conformance assessment process. ISO 9646 .

[JPTW90] V. Jensen, A. Potthof, W. Thomas, and U. Wermuth. A sort guide to the amore system (computing automata, monoids and regular expressions). Technical report, RWTH Aachen, 1990.

[Lal79] G. Lallement. Semigroups and combinatorial Applications. J. Wiley and Sons, New York, 1979.

[LPB93] G. Luo, A. Petrenko, and G.v. Bochmann. Selecting test sequences for partiallyspecified non deterministic finite state machines. Technical report, Université de Montréal, février 1993.

[NT81] S. Naito and M. Tsunoyama. Fault detection for sequential machines by transition tours. In IEEE Fault Tolerant Computing Conference, pages 238-243, , 1981.

[PG90] M. Phalippou and R. Groz. From estelle specifications to industrial test suites, using an empirical approach. In FORTE'90, Madrid, Spain, november 1990.

[Pin86] J.E. Pin. Varieties of formal languages. Oxford Univ. Press, 1986.

[SD85] K. Sabnani and A.T. Dahbura. A new technique for generating protocols test. ACM Computer Communication Review, 15(4):36-43, 1985.

[SD89] K. Sabnani and A.T. Dahbura. A protocol test generation procedure. Computer Network and ISDN Systems, 15, 1989.

[SL89] D. Sidhu and T. Leung. Formal methods for protocol testing : A detailed study. IEEE Trans. on Software Engineering, 4(15), April 1989.

[VCI89] S.T. Vuong, W.Y.L. Chan, and M.R. Ito. The uiov method for protocol test sequence generation. In $2^{\text {th }} I W P T S$, Berlin, october 1989.

[YL95] M. Yanakakis and D. Lee. Testing finite state machines: Fault detection. Journal of Computer and System Sciences, 50(2):209-227, april 1995. 